\title{
8 \\ Open Service Node For Intelligent Networks
}

\author{
Pasi Kemppainen \\ Systems Software Partners Ltd., \\ Laserkatu 6, 53850 Lappeenranta, Finland, \\ Tel. +358 53624 3272, Fax. +358 534020949 \\ E-mail:Pasi.Kemppainen@ssp.kareltek.fi
}

Olli Martikainen

Telecom Finland Ltd.

P.O.BOX 106, 00511 Helsinki, Finland,

Tel. +3582040 3503, Fax. +358 20403251

E-mail:Olli.Martikainen@tele.telebox.fi

\begin{abstract}
Intelligent Networks (IN) is a concept that supports fast and flexible creation of new services in fixed and mobile networks. In addition to generic IN services provided by telecom operators there is a need for customer oriented smaller scale services, which can be integrated to the special needs of the customer or service provider. In this paper a lightweight IN service control environment called the Open Service Node (OSN) is introduced. The OSN environment is designed for third party service creation by service providers and it can be applied in the control of fixed, mobile and broadband networks. The OSN has been developed at Telecom Finland since 1991 and it has been used in pilot projects since 1993. Systems Software Partners Ltd. is a software house specialized in IN service creation. SSP has co-developed the OSN with Telecom Finland and is currently productizing it for the international markets.
\end{abstract}

\section{INTRODUCTION}

The Open Service Node (OSN) offers an open and cost-efficient solution for creating service applications on top of Intelligent Network systems. It decreases IN service time-to-market 
through its flexible approach to service delivery from laboratory prototyping into production network. Because the OSN system is designed to be cost efficient, it is based on open standards (*IX operating system, SS\#7 protocol, TCP/IP protocols and commercial SQL database system e.g. Sybase as SDP) and open application programming interfaces for service creation.

The OSN system is based on open modular software architecture. This allows for instance the development of new interfaces to different network modules (e.g. ATM switches) and introduction of new transaction processing systems (e.g. Tuxedo) also for third parties. Furthermore, the system modularity gives the flexibility needed to implement future requirements for multiple supported INAPs, SDPs, and others. Because the OSN system is developed with C-language based protocol framework, it is highly portable to different operating systems. It is first available for HP-UX and Linux operating systems.

Service development with the OSN is at the moment done using a service script language, which consists of extended finite state automaton language. The automaton language contains the possibility to embed C-program code within the automaton script. These embedded Croutines contain, for example, methodology for logging transactions and interfacing the OSN to the Service Data Point (SDP).

This paper describes mainly the functionality of the OSN environment in the development phase $3 \mathrm{Q} / 1995$.

\section{THE OSN SYSTEM ARCHITECTURE}

The OSN system is designed to be a complete environment for developing, testing and running reliable IN service applications in the network. The architecture is shown in Figure 1. The doubled system architecture offers high availability for running IN services.

The OSN environment is designed to implement several different network configurations. The OSNs switch interface is adaptive to different switches, because the services in the OSN have built-in INAP interfaces and the services can be implemented with one or more INAP interfaces. This enables the integrity of the service management in heterogeneous networks.

Furthermore, the OSN processes are reconfigurable for new functions, so that the needed parts of the OSN SS\#7 process can be replaced e.g. with TCP/IP, ATM or GSM stacks to meet the network requirements. Also, the processes are distributable to different hosts, so that the service databases and services can be located in dedicated hosts, if needed.

The OSN system is available as

- laboratory version

- single service piloting version on the network

- doubled production network version

The OSN laboratory version runs on freeware Linux operating system and requires no expensive commercial SQL database. Therefore it is ideal for developing and testing new IN services and applications. Also, because of the OSNs open concept, the OSN is suitable for academic usage such as protocol engineering. 
The single piloting version is based on HP9000 platform and is suitable for piloting services for example in real SS\#7 network. It includes Sybase database system for fast and reliable transactions. The doubled production version (see Figure 1), which is based on HP9000 platform (with multiprocessor platform option), is a high availability version of the OSN for production network.

The estimated overall performance of the OSN is expected to be 20-30 tps, depending on traffic profile.

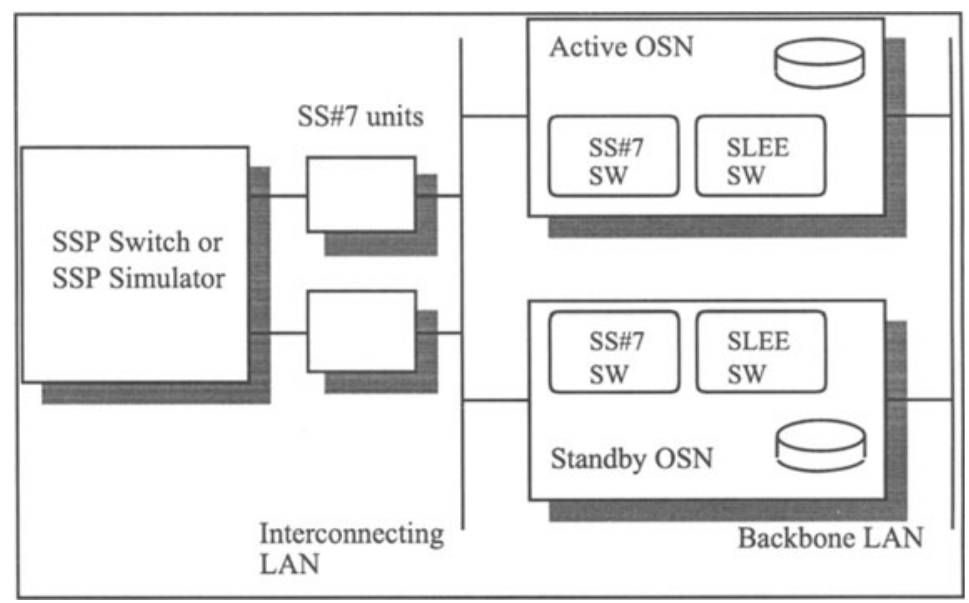

Figure 1 The OSN physical architecture.

\section{THE OSN SOFTWARE ARCHITECTURE}

The OSN software is based on CVOPS (C-language based Virtual Operating System) software architecture [1]. The CVOPS protocol framework is a portable protocol development and run-time environment which has been developed at the Telecommunications Laboratory of the Technical Research Centre of Finland (VTT). The main reason for its selection as a framework is its portability and design for protocol stack implementations.

The OSN software architecture is designed to be modular and flexible (see Figure 2). The main modules in the OSN are (the modules may include several processes):

- SS\#7 stack

- SLEE

- Scheduler

- Database query and update

- SQL database

- Service Management System server 
- Watchdog

- OSN system management

The OSN SS\#7 stack consists of front-end hardware and back-end software as shown in Figure 2. The front-end hardware consists of MTP2 Interface Units (MIUs), which are based on PC servers (see Figure 1). The interconnection to back-end is done with TCP/IP LAN and communication between systems is implemented through TCP/IP sockets.

The MIUs include signaling data link (MTP level 1) and signaling link (MTP level 2) functions. MTP level 1 protocol offers a connection to $2 \mathrm{Mbit} / \mathrm{s}$ PCM interface. MTP 2 level protocol is implemented according to CCITT blue book. The implemented architecture enables SS\#7 change-over procedures over separate links (MIUs), thus ensuring the availability of the services in the OSN. The SS\#7 back-end can use several links to increase throughput in SS\#7 network, and to give protection of link failures.

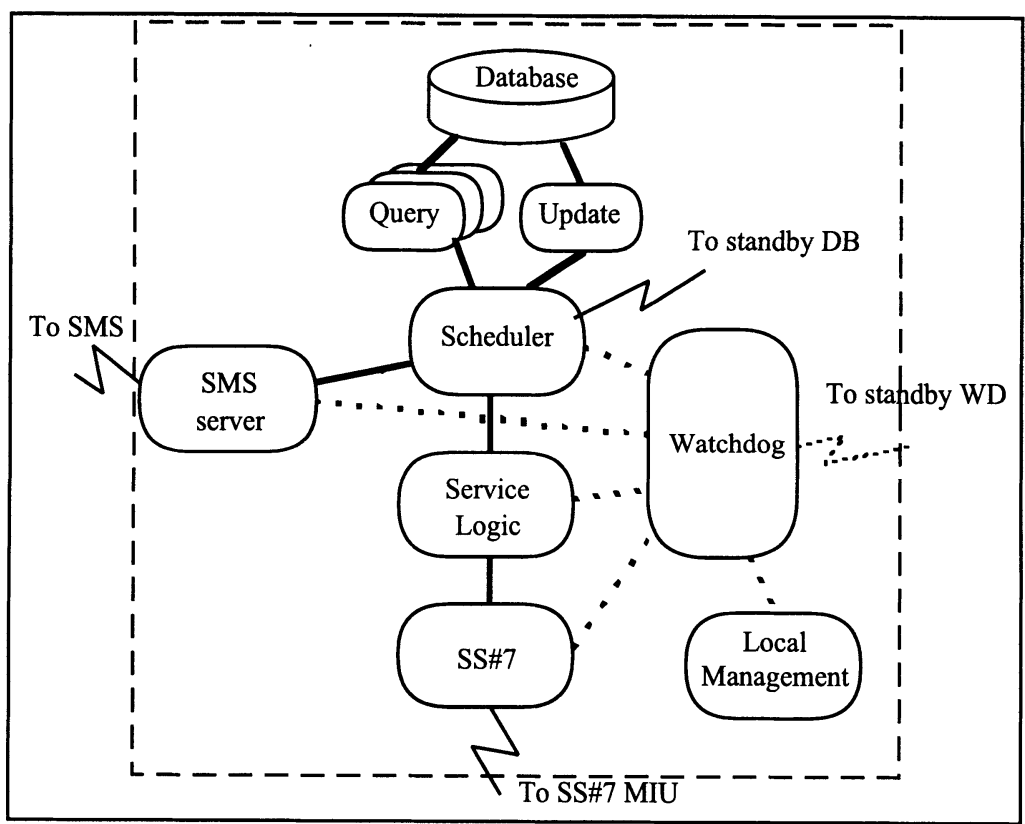

Figure 2 The OSN process architecture.

The OSN SS\#7 back-end consists of processes for MTP level 3 and SCCP and their management (see Figure 2). TCAP and INAP are implemented within the service processes, Service Logic Programs (SLPs). Current implemented INAPs are Nokia's AIN rel. 0.1 FINAP and 
ETSI Core CS-1. The modular architecture of SS\#7 and services gives the option to replace parts or all of current SS\#7 processes with different parts, if needed [2, 5].

The OSN SLEE (Service Logic Execution Environment) is based on CVOPS protocol framework. SLEE includes service processes, their management and data.

The interface between services and database is CCITT X.700 CMIP-compliant object oriented XI-interface. XI offers a programmer high abstraction of handling service parameters in database with GET, SET, CREATE, DELETE, ACTION - operations. Furthermore, the interface to operator's OSS (Operations Support System) is provided through OSN Service Management System (SMS) server XI-interface. With the XI, the database interface is possible to adapt for other database-systems, too (e.g. Oracle). The XI-interface has been developed using the previous work reported in [3].

The managed objects, methods and attributes in the OSN services' data are described in XI MIB (Management Information Base) or XIB. The execution of XI-methods are done in database query and update processes (see Figure 2). These query and update processes are used for the data access by Scheduler process.

Scheduler process is responsible for scheduling incoming database operation requests from service and system management processes in the OSN. The request operations are divided as database query requests and database update request. Scheduler offers definable priorisation for the request operations. For example, higher priorisation can be defined for services' requests than for service management requests, which are usually either large queries or batch updates to the database. Furthermore, in the doubled OSN, Scheduler controls databases' consistency. It writes data updates to both active and stand-by OSNs databases and in the system switch-over it controls that the information is equal in both databases.

Service Management System server process is a open interface to operator's OSSes. It provides operator with CMIP compliant XI-interface to service databases, provisioning of service data and access to service business functions such as billing.

The OSN is managed internally by the Watchdog process. The Watchdog starts other processes in the OSN at system reboot. The Watchdog expects a heartbeat signal from managed processes and checks that processes are alive. In error situations the Watchdog can restart failing processes or perform controlled system switch-over.

The operator management interface to the OSN processes is given through the Watchdog to make it easy for the operator to manage all processes and to reduce the number of connections between processes.

The OSN System Management is a menu driven management program in remote workstation and/or in local OSN system. System management includes e.g.

- audit processes and system usage

- $\log$ transactions and set logging levels

- configure parameters in OSN processes

- add, remove, configure services

- view service and system statistics

- perform system switch-overs and database consistency warm-ups

- perform OSN setup and shutdown 
- setup processes in OSN

OSN Management also includes Logger process for logging OSN systems' transactions in remote management workstation. It alarms operator if erroneous or fraud actions has been detected in the OSN system.

\section{SERVICE CREATION AND MANAGEMENT IN OSN}

The service creation process [4] can be supported with the OSN environment tools. In the OSN Service Creation Environment (SCE) the Service Logic Programs (SLPs) are written with CVOPS protocol framework scripting language. The scripting language is a high level, C-language like protocol EFSA (Extended Finite State Automaton) language which defines protocol states, their inputs and actions taken on inputs (see dummy example in the Appendix). The mapping between the OSN EFSA language and SDL graphical representation is seen in the Appendix. The general actions and processings can be defined as macros for higher abstraction e.g. CollectDigits, AnalyzeDigits, PromptAndCollectUserInformation and so on.

The scripting language can be easily extended with user-written C-functions for new functions and service specific actions. The automaton language is compiled into C-language using the Service Creation Environment tools. The C-code will in turn be compiled into an executable program using an ANSI compatible C-compiler.

The service creation in the OSN can be divided in four layers (see Figure 3):

- Graphical user interface for service creation (not implemented yet) which uses SDL graphical representation (see appendix) and produces EFSA language for actual service compilation (see appendix)

- High level service description language and macros which uses OSN API functions such as INAP-message coding/decoding

- OSN system level functions needed in service creation (e.g. database management, functionality of macros and new INAP interfaces)

- Operating Systems functions needed in services (e.g. string operations, socket communications and handling of exceptions and interrupts). These functions are common to all services and are used as already implemented library functions.

The OSN service creation can be started from laboratory prototyping with the switch simulation program. The tested and proofed service files can be then moved to the actual pilot and production network without any modifications to the files. This innovative approach to service creation decreases services' time-to-market and thus enhances operator's competitive advantage.

Another important feature of the service creation in the OSN is the available toolset for developing services. Toolset includes e.g. following programs: 
- A SSP switch simulation tool for emulating switch operations. It can be connected to the OSN either through TCP/IP connection or SS\#7 connection

- A telephone simulation tool which provides the user an easy and fast method to ad hoc test services. Several telephone simulators can be attached to SSP simulator and they offer both A- and B-subscriber facilities

- A traffic generator that can be used to create IN user traffic and to test service memory handling. The traffic can be defined with script language to match wanted traffic profile

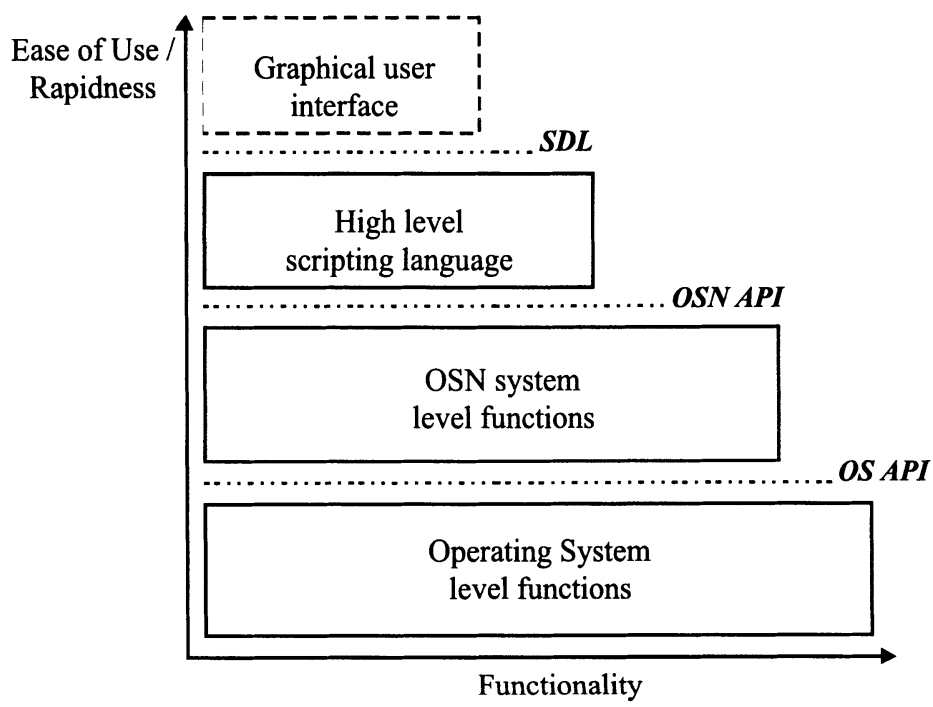

Figure 3 Layering for the OSN service creation.

- A third party commercial SQL database engine and tools for database management (Sybase 10)

- A third party commercial 4GL cross-platform UI development environment for IN service management software implementation (Uniface 4.0)

- Detailed tracing and debugging facilities

The service business functions such as billing information gathering, service data setup and service data altering are deployed through Service Management System server process. The $\mathrm{XI}$ interface is used for accessing these functions. The service management functions such as

- deployment of a new service

- removal of an existing service

- modifications to an existing service 
are done by using menu driven OSN system management program. Furthermore, the OSN system management manages the service logging, auditing and statistics.

\section{FUTURE DEVELOPMENT}

It is obvious that the one of the main future development aspects of the OSN is in graphical user interfaces (GUIs) both in service creation and in system management. The OSN SCE GUI will resemble SDL-type of graphical definition of state automatons (see dummy example in appendix). The programmer will be provided with graphical tools for service testing with a phone interface, a mass traffic generator and switch simulators. The system management GUI will include within graphical interface also statistics and clarified reports of the OSN system usage. The implemented interfaces will be X-windows and Windows NT.

Furthermore, the enhancement of the overall performance of the OSN will be examined and tested. These enhancements include e.g. more efficient disk and memory usage of the database system, multiprocessor UNIX and dedicated hosts for services and database systems.

There is also work going on to apply the OVOPS (Object Virtual Operations System) environment for service application development [6]. Another future development area will be the broadband intelligence for ATM-networks, which has been discussed in detail in papers $[7,8$, 9].

Because the OSN is very suitable for different network configurations, it is already used in various pilot projects in Telecom Finland and Technical Research Center of Finland (VTT). For example, the OSN system will be available in the EU ACTS Mobile National Host in Otaniemi, Espoo to provide mobility management in the UMTS network trials.

\section{CONCLUSIONS}

In this paper we have introduced a lightweight service control system called the Open Service Node (OSN) which can be applied in the control of fixed, mobile and broadband networks. Because the OSN system has been designed for differentiated services, the main objectives in the development have been

- cost effective IN service controlling system

- adaptation to different network and system configurations

- open interfaces for third party developers

- flexible and modular software architecture

- scalable and portable system

- reliable high availability doubled system

- TMN-compliant OSS interface. 


\section{ACRONYMS}

\begin{tabular}{|c|c|}
\hline $4 G L$ & 4:th Generation Language \\
\hline$A C T S$ & Advanced Communications Technologies and Services \\
\hline AIN & Advanced Intelligent Network \\
\hline ATM & Asynchronous Transfer Mode \\
\hline$A P I$ & Application Program Interface \\
\hline CMIP & Common Management Information Protocol \\
\hline CMIS & Common Management Information Service \\
\hline CVOPS & C-language based Virtual Operating System \\
\hline & Capability Set \\
\hline$D E C T$ & Digital European Cordless Telephone \\
\hline EFSA & Extended Finite State Automaton \\
\hline GSM & Global System for Mobile communications \\
\hline GUI & Graphical User Interface \\
\hline IN & Intelligent Network \\
\hline INAP & Intelligent Network Application Part \\
\hline IP & Intelligent Peripheral \\
\hline$L A N$ & Local Area Network \\
\hline$M I B$ & Management Information Base \\
\hline$M I U$ & MTP Interface Unit \\
\hline MTP & Message Transfer Part \\
\hline OSN & Open Service Node \\
\hline OSS & Operations Support System \\
\hline$P C M$ & Pulse Code Modulation \\
\hline$S C C P$ & Signaling Connection Control Point \\
\hline$S C E$ & Service Creation Environment \\
\hline$S C P$ & Service Control Point \\
\hline SLEE & Service Logic Execution Environment \\
\hline$S \dot{L} P$ & Service Logic Program \\
\hline$S M S$ & Service Management System \\
\hline$S N$ & Service Node \\
\hline$S Q L$ & Structured Query Language \\
\hline SS\#7 & Signalling System Number 7 \\
\hline SSP & Service Switching Point \\
\hline TCAP & Transaction Capabilities Application Part \\
\hline TCP/IP & Transmission Control Protocol/ Internet Protocol \\
\hline UMTS & Universal Mobile Telecommunications System \\
\hline & $\mathrm{X}$ Interface \\
\hline
\end{tabular}

\section{REFERENCES}

[1] J. Malka, E. Ojanperä, "Reference Manual for CVOPS 4.0", Technical Research Centre of Finland, Telecommunications Laboratory, 1992, 1 - 107.

[2] O. Martikainen, V. Naoumov and K. Samouylov, Signalling System No. 7 Portable Software Implementation, St. Petersburg Regional International Teletraffic Seminar, 1520.6.1993, $344-348$. 
[3] J. Airaksinen, O. Martikainen, J. Sonninen and H. Töhönen, UPT Service Management, International Workshop on Intelligent Networks, Lappeenranta 10.8.1993, 1 - 14.

[4] T. Karttunen and O. Martikainen, Open Intelligent Network Application Development, Proceedings of the International Symposium on Network Information Processing Systems, Sofia 12-14.10.1993, 77-90.

[5] O. Martikainen, V. Naoumov and K. Samouylov, Portable Intelligent Network Software Implementation, Proceedings of the International Symposium on Network Information Processing Systems, Sofia 12-14.10.1993, 91-96.

[6] O. Martikainen, P. Puro and J. Sonninen, The OVOPS Environment for IN Applications, IN '94 Workshop, Heidelberg, Germany, 24 - 26.5.1994, 1 - 14.

[7] K. Molin, O. Martikainen, Broadband Intelligent Network Project, Workshop on Intelligent Networks, 8.-9.8.1994, Lappeenranta, 253 - 263.

[8] O. Martikainen, T. Karttunen, V. Naoumov, K. Samuylov, Comparison of Broadband Intelligent Network Architectures, Workshop on Intelligent Networks, 8.-9.8.1994, Lappeenranta, 265 - 283.

[9] O. Martikainen, J. Lipiäinen, K. Molin, Tutorial on Intelligent Networks, Workshop on Intelligent Networks, 8.-9.8.1994, Lappeenranta,1-88.

\section{BIOGRAPHY}

Pasi Kemppainen received his M.Sc. in telecommunications in 1995. He has worked in the area of data communications and telecommunications since 1991 concerning EDI, X.400, telecommunications and internet technologies in various companies and also as a free-lancer. Currently he is Development Manager in Systems Software Partners (SSP) Ltd. and is specialized in software technologies for Intelligent Network service control and creation. Also, he works as a part-time assistant in Helsinki University of Technology for telecommunications cources.

Olli Martikainen, Ph.D., from Helsinki University and M.Sc. from Helsinki University of Technology. He has been doing research in several positions in Helsinki University of Technology (1976 - 1982), Oxford University (1980 - 1981) and Technical Research Centre of Finland (1982 - 1985). Later he has been R\&D manager at Nokia Electronics (1985 - 1986), department manager at Nokia Research Centre (1986 - 1988), professor and head of Datacommunications Institute at Lappeenranta University of Technology (1988 - 1989) and professor at Technical Research Centre (1989 - 1991). Since 1991 he has been research director at Telecom Finland. Currently he is vice president, R\&D, at Telecom Finland Ltd., and professor at Helsinki University of Technology. His main areas of interest are telecommunication software methods and tools, network architectures, performance analysis and new industrial and economic structures in telecommunications. 


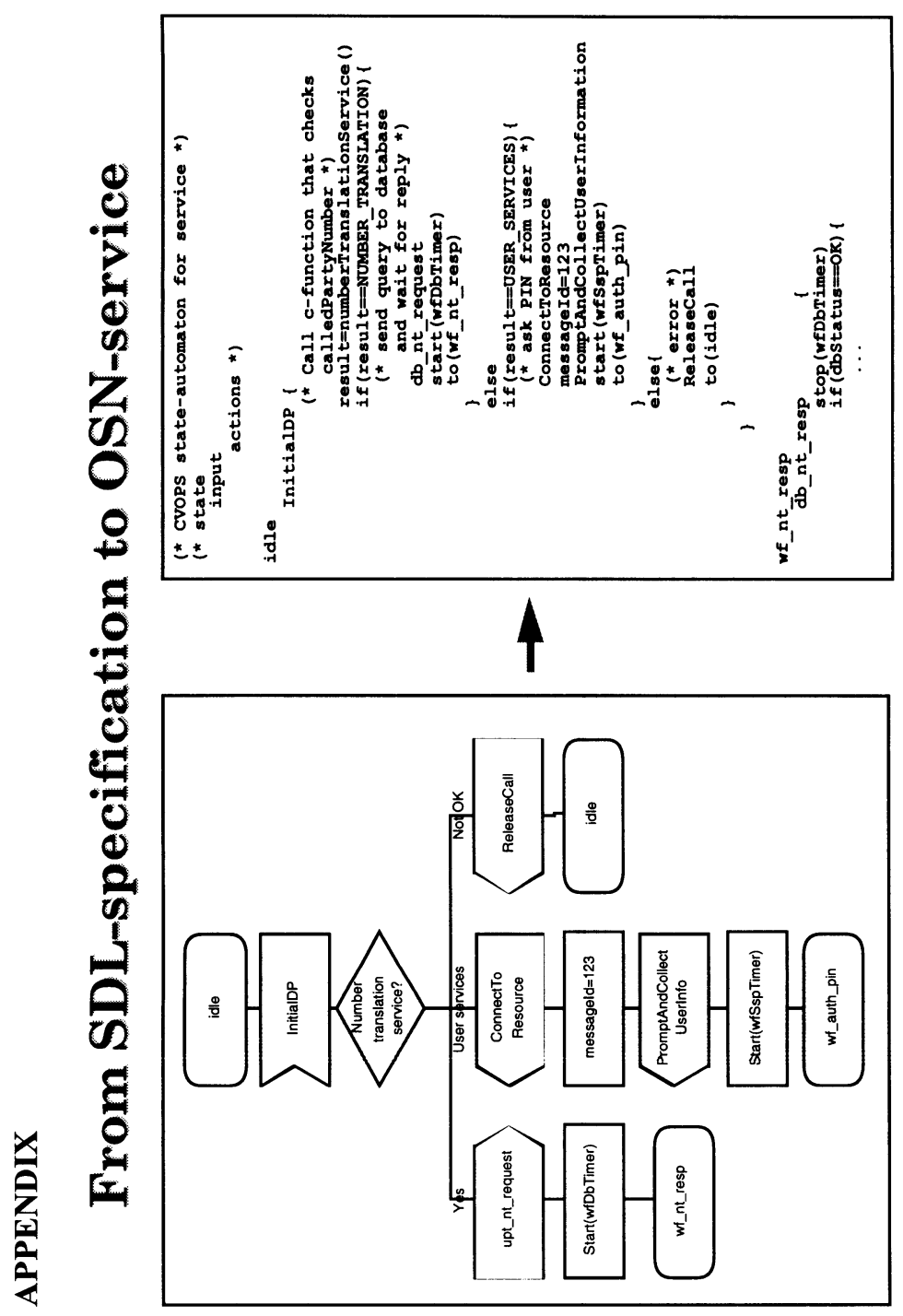

